\title{
USE A MODIFIED RING METHOD TO PREDICT DRAPE PROPERTIES IN DRAPERY FABRICS
}

\author{
Enric Carrera-Gallissà ${ }^{1}$, Xavier Capdevila $^{2}$, Josep Valldeperas ${ }^{1}$ \\ ${ }^{1}$ Institut d'Investigació Tèxtil i Cooperació Industrial de Terrassa, Universitat Politècnica de Catalunya. C/ Colom, 11. 08222-Terrassa. Spain; \\ enric.carrera@upc.edu, valldeperas@intexter.upc.edu \\ ${ }^{2}$ Departament de Ciència dels Materials i Enginyeria Metalılúrgica (Secció d’Enginyeria Tèxtil).Universitat Politécnica de Catalunya. C/ Colom, \\ 1. 08222-Terrassa. Spain; francisco.javier.capdevila@upc.edu
}

\section{Abstract:}

Seven drape indicators and four of the parameters assessed in the UPC ring test were determined by using canonical correlations in 15 commercial drapery fabrics of variable composition, aerial weight and weave type. A regression equation relating drape ratio $(\% D R)$ and parameter $h$ in the ring test was thus established. Based on the results, the ring test provides a useful tool for the routine industrial assessment of drape in finished drapery fabrics without the need for additional equipment to determine the drape.

\section{Keywords:}

Drape, ring method, drapery fabrics, canonical correlatio

\section{Introduction}

Drape is an aesthetic property that can dictate consumers' fabric buying decisions; hence, its accurate assessment is of especial industrial, economic and commercial importance. Ever since the 1960s, drape has routinely been determined with a Cusick drape meter.[4] Tests are relatively slow to perform and require some skill in the preparation and handling of specimens in order to avoid measurement errors. Also, they entail using a balance and special calibrated paper. However, the advent of digital electronics has allowed the human eye to be replaced with a digital camera in order to capture drape shadow in Cusick meters. Also, the ability to process the digital information thus acquired with imaging software has facilitated careful examination of the shadow geometry and elucidation of the drape phenomenon. Despite these technological advances, the test continues to be time-consuming and requires the use of specific equipment.

\section{Aims}

The primary aim of this study was to check whether the modified ring test method[2] is an effective alternative to the
Cusick drape meter with a view to the industrial assessment of fabric drape characteristics. The proposed method, the most salient advantage of which is that it can be implemented with a conventional dynamometer typically used by the textile industry and a small ring available in most mechanical workshops, would be exclusively used for quality control of fabric finishing properties.

\section{Material and methods}

The Cusick drape meter was operated as follows: a circular specimen of fabric $30 \mathrm{~cm}$ in diameter was placed on an $18-\mathrm{cm}$ rigid metal disc; as a result, the specimen portion not directly resting on the disc fell freely as a function of its drape. A light source aligned with the focus of a parabolic mirror was placed right below the center of the specimen to project a light beam downwards. The beam was reflected upwards by the mirror and intercepted the specimen. As a result, the specimen projected its drape shadow onto a paper ring located in a parallel plane above it. Figure 1 shows a current drape meter design.

Measuring drape entailed drawing the drape shape by tracing the contour of the drape shadow on the paper ring and cutting
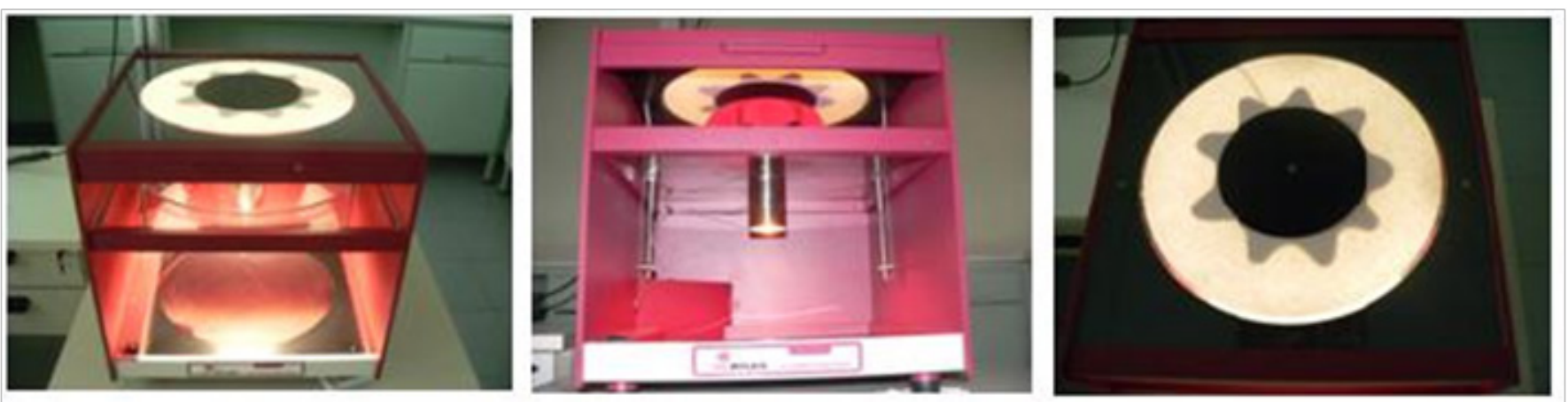

Figure 1. Current design of the Cusick drape meter (Photo: Department of Textile and Paper Engineering, UPC) 
it out. The portion of paper containing the shadow projected by the fabric drape was then weighed and the resulting weight $W_{1}$ divided into the weight of the whole paper ring $W_{2}$ to obtain the drape ratio \%DR.

$$
\% \mathrm{DR}=\left(\mathrm{W}_{1} / \mathrm{W}_{2}\right) \times 100
$$

This test conforms to British standard BS 5058, 1973 ("Method for the assessment of drape of fabrics").

Each of the four specimens was evaluated on both the face and the reverse, and the two values thus obtained were used to calculate a mean.

Measurements were made by using a Cusick drape meter equipped with a computer-interfaced CCD camera mounted at the top. The equipment (Fig. 2) belongs to the Textile Physics Laboratory of the Department of Textile and Paper Engineering of the Polytechnic University of Catalonia (UPC).

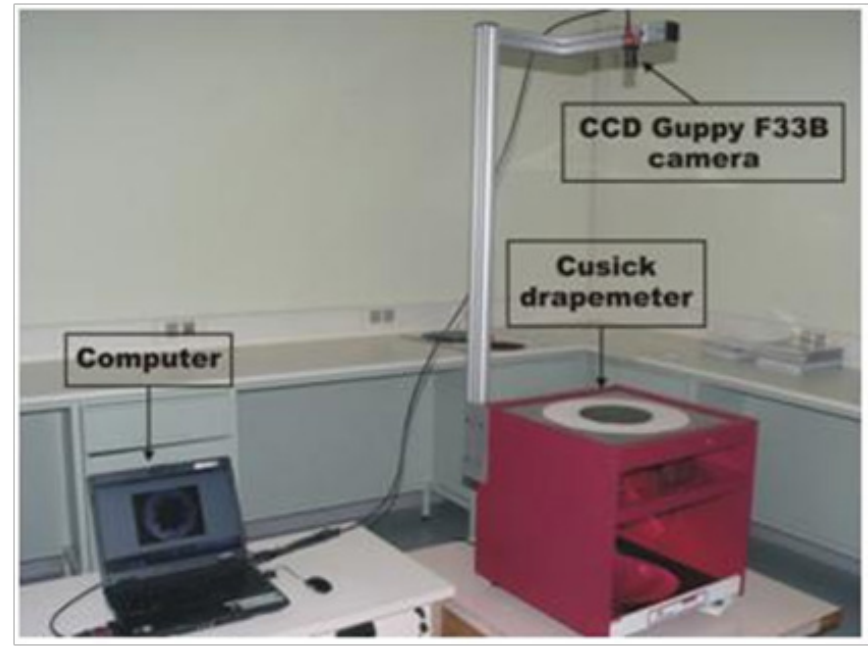

Figure 2. Digital UPC drape meter of the Department of Textile and Paper Engineering of UPC

A total of 4 specimens $30 \mathrm{~cm}$ in diameter of each fabric type and an 18-cm support disc were used. Each specimen was examined at three different rotation angles $\left(0,90\right.$ and $\left.135^{\circ}\right)$, both on the face and on the reverse. The drape value assigned to each fabric was thus the arithmetic mean of 24 determinations. All specimens were allowed to adjust to a standard atmosphere prior to testing.

The specific drape indicators determined were as follows:

a) Drape ratio, \%DR. ${ }^{[4]}$ As stated above, \%DR is the percent fraction of the paper ring area spanned by the projection of the free-falling specimen. \%DR can range from $0 \%$ (lowest drape) to $100 \%$ (highest drape).

b) Fold number, $\mathrm{FN},{ }^{[3]}$ which is the visually determined number of maxima in the drape profile of a specimen.

c) Mean fold height, $\mathrm{FH},{ }^{[6]}$ which is the mean of the fold heights measured from the line used to determine FW (see Fig. 3).

$$
F H=\sum_{i=1}^{n} \frac{F H i}{n}
$$

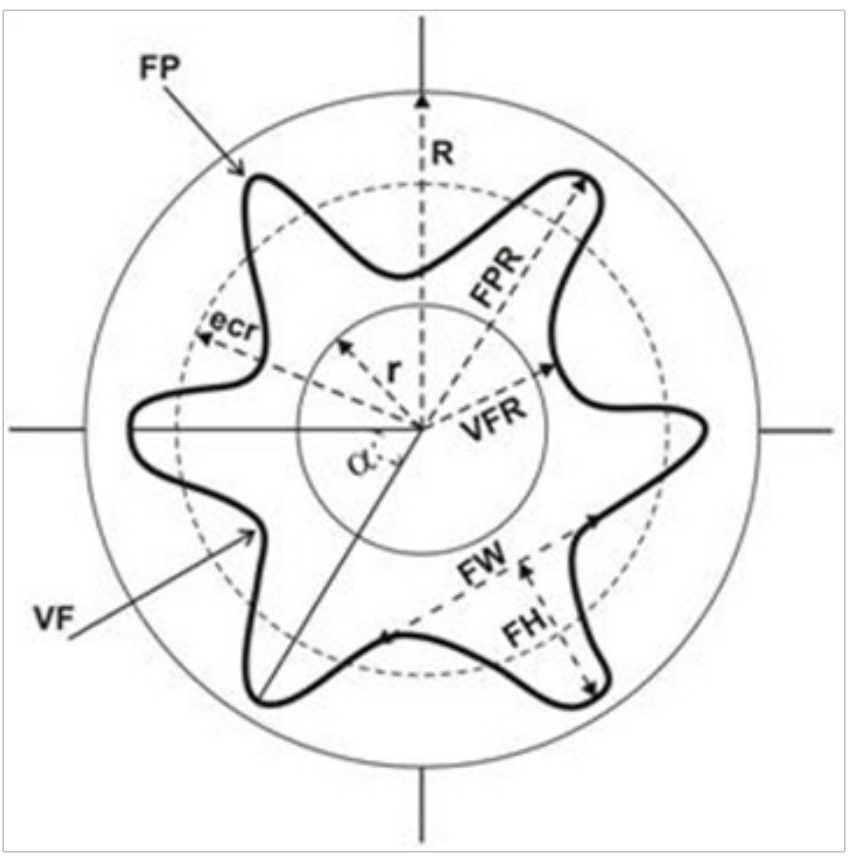

Figure 3. FP fold peak, VF fold valley, FPR fold peak ratio, VFR fold valley ratio, FW fold width, FH fold height, $r$ support disc radius, $R$ specimen radius, rce circumference radius, $\alpha=$ angle between consecutive folds.

d) Variability in fold severity, VS. ${ }^{[8]}$ This is the standard deviation of severity in the folds of each specimen and a measure of unevenness in the drape shape and severity. It accounts for uniformity in the folds but not for their shape. The drape fold profile is transformed into a coordinate system with radii $(\mathrm{mm})$ on the $y$-axis and degrees or the perimeter of the unfallen specimen on the $x$-axis. The mean fold severity is calculated as the arithmetic mean of the ratio of height of each node $(h)$ to its width $(b)$ (see Fig. 4 ).

$$
V S=\sqrt{\frac{\sum_{i=1}^{n}(M N S \mathrm{i}-\overline{\mathrm{MNS}})^{2}}{n-1}}
$$

e) Drape unevenness, \%DU, ${ }^{[1]}$ which is the coefficient of variation of the angle between consecutive folds. This is a measure of drape symmetry in each specimen, but not of drape shape. It is expressed as a percentage and can range from $0 \%$ (maximum symmetry) to $100 \%$ (minimum symmetry).

$$
D U \%=\frac{\sqrt{\frac{\sum_{i=1}^{n}(\alpha \mathrm{i}-\bar{\alpha})^{2}}{n-1}}}{\bar{\alpha}} \cdot 100
$$

f) Folding distribution, \%Gp, ${ }^{[5]}$ which is the coefficient of variation of fold peak radius (FPR) and a measure of variability in fold shape and symmetry, in each specimen. 


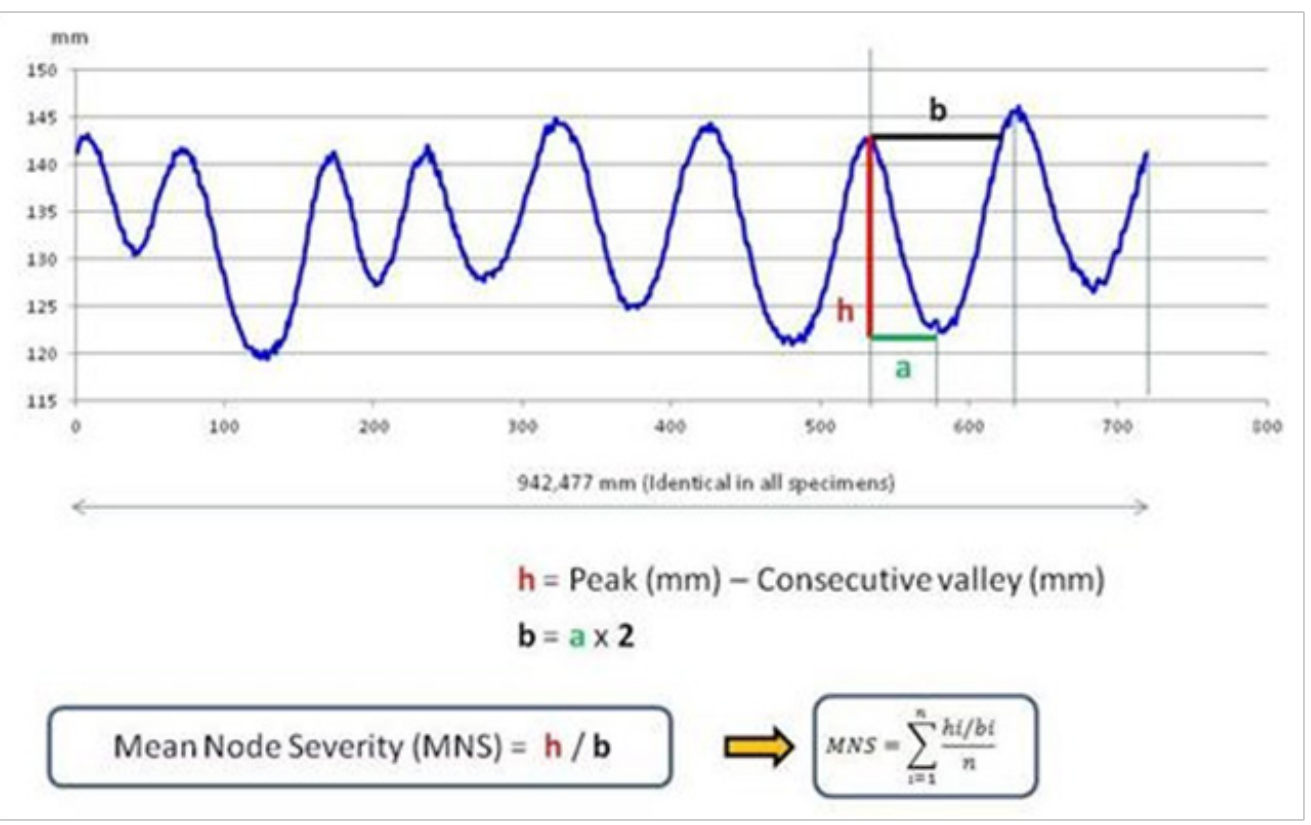

Figure 4. Procedure used to calculate Mean Node Severity
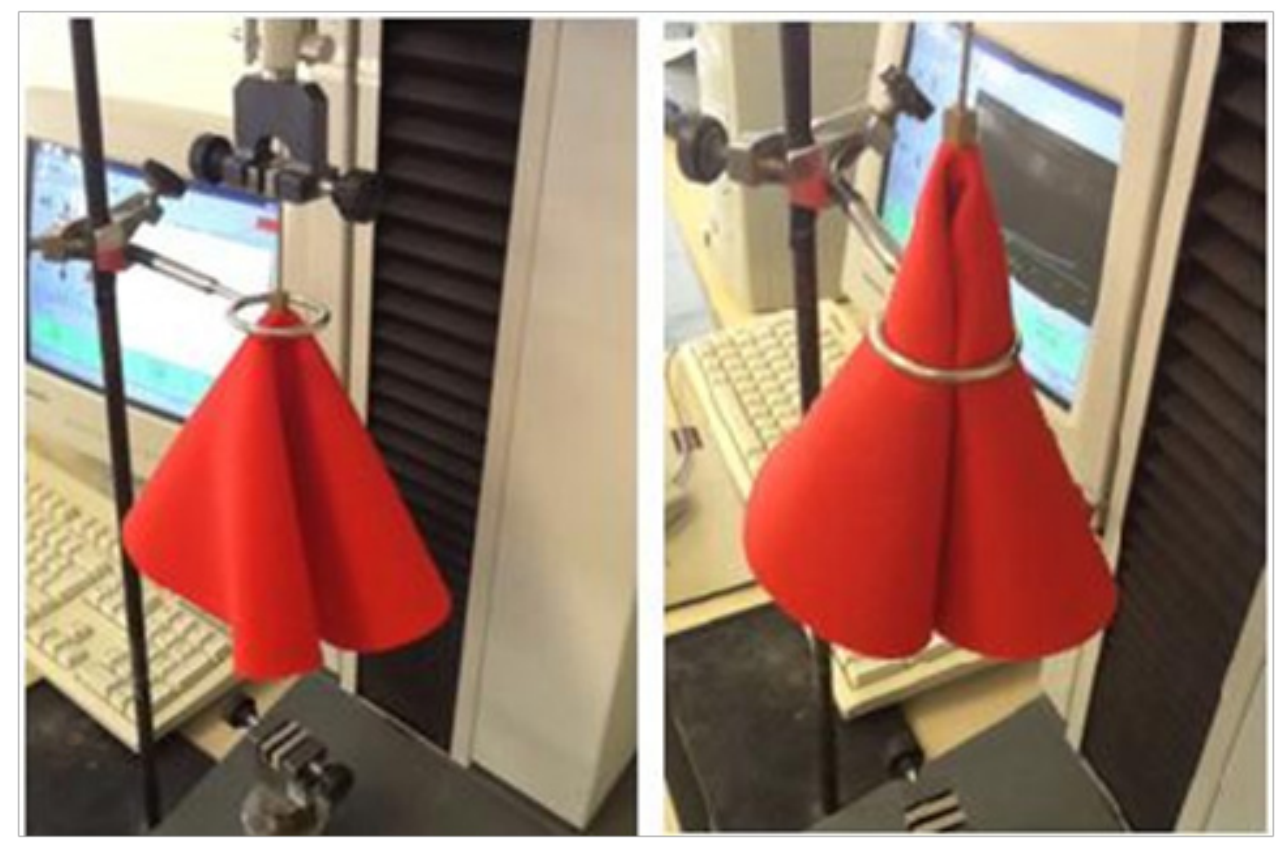

Figure 5. Sequence of the UPC ring method. (Left) Start of the test. (Right) During the test

$$
\% G p=\frac{\sqrt{\frac{\sum_{i=1}^{n}(F P R \mathrm{i}-\overline{F P R})^{2}}{n-1}}}{\overline{F P R}} \cdot 100
$$

g) Slimness or geometric isotropy ratio, D/O.[6] According to the proponents of this indicator, the drape profile of a fabric is simply a periodic function that can be transformed into a Fourier series to determine the frequency of the dominant wave. $\mathrm{D} / \mathrm{O}$ is the ratio of the area under the dominant curve to that of the drape shadow.

$$
\frac{D}{O}=\frac{\text { Area of the Fourier transformed shape }}{\text { Area of the original captured drape image }}
$$

A near-unity ratio indicates that the original curve (drape projection) is very similar to the dominant weight profile (a perfect sine function). As a result, the original curve will render a highly regular, symmetric, even profile. Ultimately, $\mathrm{D} / \mathrm{O}$ accounts for drape shape and symmetry, and hence for geometric isotropy. The more $\mathrm{D} / \mathrm{O}$ departs from unity, the less isotropic (or more anisotropic) will be the drape shape. D/O can range from 0 (lowest isotropic geometry) to 1 (highest isotropic geometry).

The previous indicators can be used to quantify various aspects of drape. Thus, \%DR and FN measure drape intensity; FH and VS account partly for drape shape, severity or fold depth (i.e., profile roughness); and \%DU, \%Gp and D/O explain profile unevenness and geometric isotropy. 
In the variant named "UPC-Ring method", developed by the authors, ${ }^{[2]}$ the test head is a 4-mm thick polished stainless-steel circular ring held by an external support. The ring is placed at a pre-calibrated position under the vertical of the upper jaw of a conventional dynamometer. In this work, we used circular fabric specimens $30 \mathrm{~cm}$ in diameter. This specimen dimension was used as the starting point to identify a universal ring diameter allowing a wide range of commercial fabrics to be readily extracted without the need to apply much force on the specimens. A ring of $36 \mathrm{~mm}$ inner diameter was found to fulfil these requirements.

The tests ran as follows: each specimen was pierced with a rigid metal needle $80 \mathrm{~mm}$ long and $2 \mathrm{~mm}$ thick at its geometric center. The specimen was retained at its lower end by an inverted T-piece. The metal needle was held by the dynamometer upper jaw (see Fig. 5). Starting the dynamometer caused its upper jaw to fall at $100 \mathrm{~mm} / \mathrm{min}$. Because the fabric was not yet in contact with the ring, the extraction force-displacement force curve for the jaw had a zero slope. When the specimen touched the ring (Fig. 6, image 3), the curve acquired a slope dependent on the extraction force. The test was finished when the whole specimen had passed through the ring.
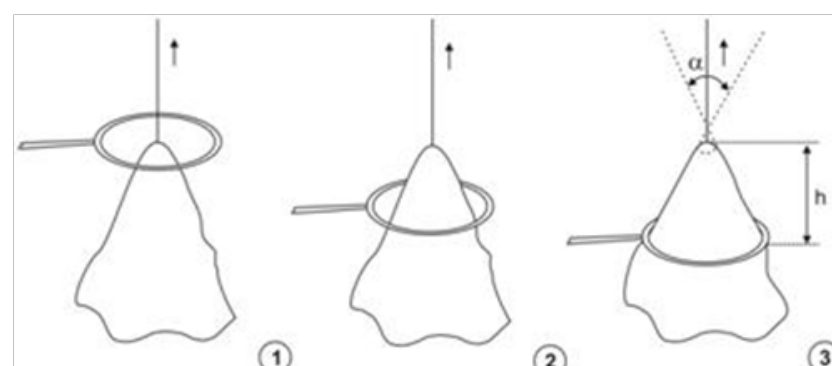

(3)

Figure 6. Stages of the UPC ring test

Table 1. Characteristics of the woven drapery fabrics studied
The dynamometer recorded the extraction force-displacement curve for the specimen. This allowed us to calculate the distance $h$ between the start of the test and the time the specimen came into contact with the ring (Fig. 7); that point is shown in Fig. 6 , image 3 . The recording also allowed the calculation of the maximum extraction force and its associated displacement.

The proposed method was used to examine 4 circular specimens of $30 \mathrm{~cm}$ diameter on both the face and the reverse. This provided 8 mean values that were used to calculate the following parameters:

the maximum extraction force $\left(F_{\text {max }}\right)$;

the displacement needed to reach it $\left(D F_{\text {max }}\right)$;

the distance $h$ between the start of the test and the point where the specimen first touched the ring (Fig. 6 image 3); and the drape angle $(\alpha)$, which was obtained trigonometrically from $h$ and the ring radius (Fig. 6, image 3).

All tests were performed at the Textile Physics Laboratory of the Department of Materials Science (Textile Engineering Section) of the Polytechnic University of Catalonia and all specimens were allowed to adjust to the standard atmosphere of the laboratory before measurement.

Table 1 summarizes the characteristics of the 15 woven drapery fabrics studied.

\section{Results}

We examined the linear correlations of drape indicators to the parameters of the ring method. Multicollinearity and the associated problems in interpreting correlation coefficients was avoided by considering two groups of variables with internal

\begin{tabular}{|c|c|c|c|}
\hline Reference & Composition & Aerial weight $\left(\mathbf{g} / \mathbf{m}^{\mathbf{2}}\right)$ & Wave type \\
\hline 1 & WO/PAC 60/40 & 447.41 & Double cloth \\
\hline 2 & PES/CV/ELAST 64/31/5 & 371.50 & Serge \\
\hline 3 & PES/RAI/ELAST 78/18/4 cloth \\
\hline 4 & WO/PA 90/10 & 341.33 & Double cloth \\
\hline 5 & CO/WO/PA 76/19/5 & 333.26 & Satin \\
\hline 6 & WO 100\% & 309.14 & Serge \\
\hline 7 & PES/RAI/ELAST 78/17/5 & 299.41 & Crepe \\
\hline 8 & WO/ELAST 99/1 & 279.08 & Taffeta \\
\hline 9 & PES/RAI/ELAST 65/31/4 & 232.16 & Serge \\
\hline 10 & WO 100\% & 221.17 & Serge-Herring bone \\
\hline 11 & WO/PES 60/40 & 209.00 & Serge \\
\hline 12 & WO 100\% & 199.08 & Taffeta \\
\hline 13 & CV/WO/PES 43/34/24 & 191.08 & Serge \\
\hline 14 & PES/LLI 55/45 & 171.25 & Serge \\
\hline 15 & WO/LL/CO/PA 36/32/16/16 & 163.58 & 157.71 \\
\hline
\end{tabular}


Table 2. Correlation matrix for drape indicators. The first and second figure in each cell corresponds to $R$ and the $P$-value, respectively.

\begin{tabular}{|c|c|c|c|c|c|c|}
\hline FN & FH & DU\% & Gp\% & VS & D/O & \\
\hline \multirow[t]{6}{*}{$\begin{array}{c}-0.888 \\
0.000\end{array}$} & $\begin{array}{c}-0.041 \\
0.885\end{array}$ & $\begin{array}{l}0.052 \\
0.853\end{array}$ & $\begin{array}{c}-0.586 \\
0.022\end{array}$ & $\begin{array}{l}0.183 \\
0.514\end{array}$ & $\begin{array}{l}0.595 \\
0.019\end{array}$ & \%DR \\
\hline & $\begin{array}{c}-0.041 \\
0.885\end{array}$ & $\begin{array}{c}-0.313 \\
0.256\end{array}$ & $\begin{array}{l}0.244 \\
0.381\end{array}$ & $\begin{array}{c}-0.340 \\
0.215\end{array}$ & $\begin{array}{c}-0.236 \\
0.398\end{array}$ & FN \\
\hline & & $\begin{array}{l}0.708 \\
0.003\end{array}$ & $\begin{array}{l}0.700 \\
0.004\end{array}$ & $\begin{array}{l}0.448 \\
0.094\end{array}$ & $\begin{array}{c}-0.692 \\
0.004\end{array}$ & FH \\
\hline & & & $\begin{array}{l}0.539 \\
0.038\end{array}$ & $\begin{array}{l}0.587 \\
0.021\end{array}$ & $\begin{array}{c}-0.623 \\
0.013\end{array}$ & \%DU \\
\hline & & & & $\begin{array}{l}0.192 \\
0.492\end{array}$ & $\begin{array}{c}-0.826 \\
0.000\end{array}$ & $\% G p$ \\
\hline & & & & & $\begin{array}{c}-0.225 \\
0.420\end{array}$ & VS \\
\hline
\end{tabular}

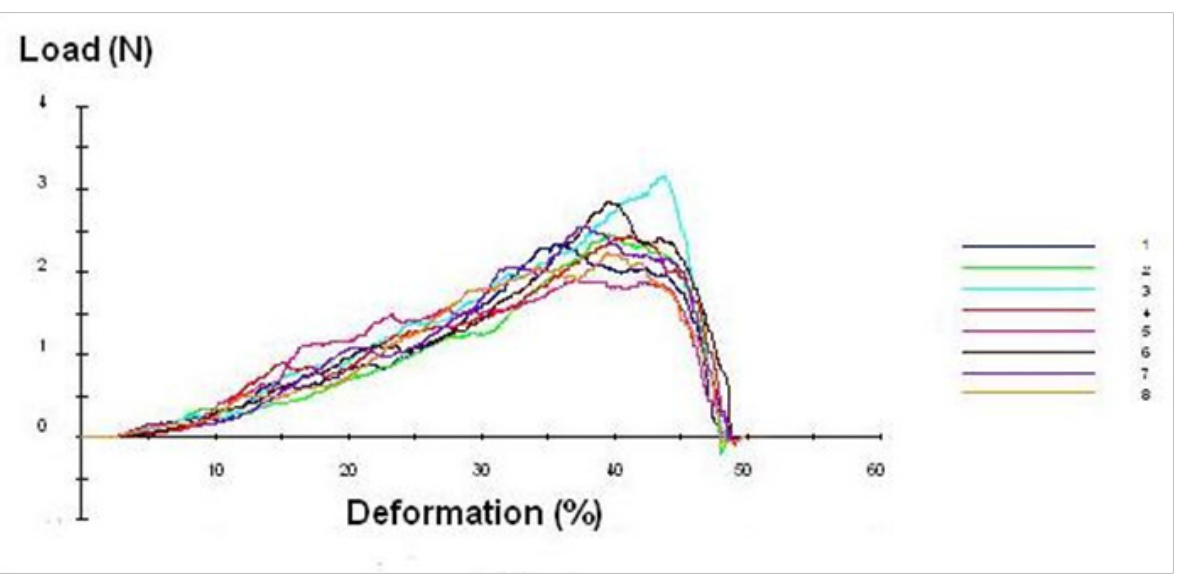

Figure 7. Fabric extraction force-displacement curve

correlations less than 0.7 . One group was formed by four drape indicators for the fabrics (\%DR, \%DU, \%Gp and VS) and the other by two parameters of the UPC ring method ( $h$ and $F_{\text {max }}$ ). The corresponding correlation matrices are shown in Tables 2 and 3.

Canonical correlation analysis was used to identify a combination of variables in each group (canonical variables) leading to a high proportion of shared variance (coefficient of determination).

Table 4 shows the first two canonical variables; only the first, however, exhibited statistically significant correlation at the $95.0 \%$ confidence level. The correlation equations for the first pair of canonical variables were as follows:

$$
\begin{gathered}
U 1=(-0,901 \cdot D R \%)-(0,166 \cdot D U \%)+(0,162 \cdot G p \%)+(0,064 \cdot V S) \\
V 1=(-0,929 \cdot h)-(0,099 \cdot \text { Fmax })
\end{gathered}
$$

Table 3. Correlation matrix for parameters of the UPC ring test. The first and second figure in each cell corresponds to $R$ and the $P$-value, respectively.

\begin{tabular}{|c|c|c|c|}
\hline $\boldsymbol{\alpha}$ & Fmax & DFmax & \\
\hline-0.998 & -0.685 & -0.098 & $\mathrm{~h}$ \\
0.000 & 0.005 & 0.727 & \\
\hline & 0.705 & 0.127 & $\mathbf{\alpha}$ \\
& 0.003 & 0.652 & Fmax \\
\hline & & 0.738 & \\
\hline
\end{tabular}

where the variables have been normalized by subtraction of their means and subsequent division into their standard deviations.

Figure 8 is a plot of the two groups of variables (drape- and ring-related) based on the canonical variables $U_{1}$ and $V_{1}$. The figure illustrates the strength of the relationship: 0.95 .

Table 4. Canonical correlations between variables

\begin{tabular}{|c|c|c|c|c|c|c|}
\hline Number & $\mathbf{R}^{2}$ & Correlation & Lambda & c2 & DF & P-value \\
\hline 1 & 0.905 & 0.951 & 0.0622 & 29.151 & 8 & 0.000 \\
\hline 2 & 0.343 & 0.586 & 0.656 & 4.420 & 3 & 0.219 \\
\hline
\end{tabular}


Table 5. Figures of merit of the analysis of variance

\begin{tabular}{|c|c|c|c|c|c|}
\hline Source & Sum of squares & GI & Mean square & F-ratio & P-value \\
\hline Model & $1.78121 \mathrm{E} 7$ & 1 & $1.78121 \mathrm{E} 7$ & 160.47 & 0.0000 \\
\hline Residual & $1.44298 \mathrm{E} 6$ & 13 & $110,998.00$ & & \\
\hline Total (Corr.) & $1.92551 \mathrm{E} 7$ & 14 & & & \\
\hline
\end{tabular}

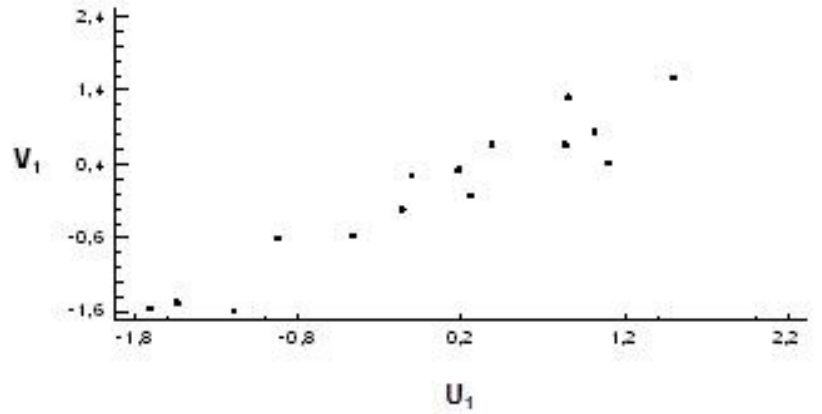

Figure 8. Plot of the canonical variables $V_{1}$ and $U_{1}$

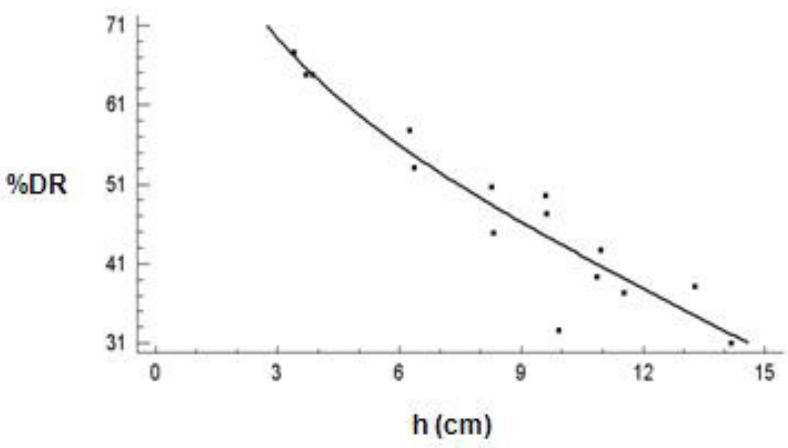

Figure 9. Regression plot of \%DR versus $h$

The magnitude of the coefficients of the previous equations (canonical weights) was a measure of the relative contribution of each natural variable to the canonical variables. The coefficient of \%DR in the equation for $U_{1}$ markedly exceeded those of the other indicators, and so did the coefficient of $h$ in relation to $F_{\text {max }}$ in the equation for $V_{1}$. Therefore, $U_{1}$ and $V_{1}$ were essentially accounted for by \%DR and $h$, respectively.

These equations can be used for predictive purposes. However, it is more practical-and statistically robust - in this respect to regress \%DR on $h$. The equation for the fitted model (Fig. 9) was

$$
\% D R=\sqrt[2]{(7.499,13-2.439,85 \cdot \ln (h)}
$$

Since the $P$-value in the ANOVA table (Table 5 ) was less than $0.05, \% \mathrm{DR}$ and $h$ were statistically significantly related at the $95.0 \%$ confidence level.

$R=-0.961$

$R^{2}=92.506 \%$

$R^{2}($ adjusted $)=91.929 \%$

Durbin-Watson statistic $=1.728(P=0.2820)$

Lag 1 autocorrelation of residuals $=0.054$
Based on the $R^{2}$ value obtained, the fitted model explained $92.506 \%$ of the variability in $\% \mathrm{DR}$. The correlation coefficient, $R=-0.962$, indicates a relatively close relationship between the variables.

The Durbin-Watson statistical method examines residuals to identify potential significant correlations based on the sequence in which they occur in the data file. Since $P$ was greater than 0.05 , there was no sign of serial autocorrelation in the residuals at the $95.0 \%$ confidence level.

\section{Conclusions}

A regression equation relating \%DR with parameter $h$ in the ring test was established. Based on the results, the ring test can be used at the industrial level to routinely determine drape in finished drapery fabrics without the need for additional, dedicated equipment to measure drape.

\section{References}

[1] Al-Gaadi, B., Göktepe, F. and M. Halász. A new method in fabric drape measurement and analysis of the drape formation process. Textile Research Journal, 2012, vol. 82 (5), pp. 502-512.

[2] Carrera-Gallissà, E., Capdevila, X. and J. Valldeperas. Correlation analysis between a modified ring method and the FAST system. Journal of Engineered Fibers and Fabrics, 2014, vol. 9 (1), pp.131-140.

[3] Chu, C.C., Platt, M.M. and W. Hamburger. Investigation on the factors affecting the drapeability of fabrics. Textile Research Journal, 1960, vol. 20, pp. 66-67.

[4] Cusick, G. E. (1962). "A Study of Fabric Drape". PhD thesis. University of Manchester, 1962.

[5] Jevsnik, S. and J. Gersak. Modelling the fused panel for a numeric simulation of drape. Fibres \& Textiles in Eastern Europe, 2004, vol. 12, pp. 47-52.

[6] May-Plumlee, T., Eischen, J. Kenkare, N.S., and P. Pandurangan. (2003). "Evaluating 3D Drape Simulations: Methods and Metrics", in Proceedings of International Textile Design and Engineering Conference (INTEDEC), Edinburgh, Scotland, September 22-24, 2003.

[7] Park, C.K., Kim, S. and W.R. Yu. Quantitative fabric drape evaluation system using image processing technology (Part 1: Measurement system and geometric model). Journal of Testing Evaluation, 2004, vol. 32 (2), pp. $131-$ 137.

[8] Robson, D. and C.C. Long. Drape Analysis using image techniques. Clothing and Textiles Research Journal, 2000, vol. 18 (1), pp. 1-8. 